\title{
Zero-error Coordinated Tracking of Multiple Lagrange Systems Using Continuous Control
}

\author{
Ziyang Meng, Dimos V. Dimarogonas, and Karl H. Johansson
}

\begin{abstract}
In this paper, we study the coordinated tracking problem of multiple Lagrange systems with a time-varying leader's generalized coordinate derivative. Under a purely local interaction constraint, i.e., the followers only have access to their local neighbors' information and the leader is a neighbor of only a subset of the followers, a continuous coordinated tracking algorithm with adaptive coupling gains is proposed. Tracking errors between the followers and the leader are shown to converge to zero. Then, we extend this result to the case when the leader's generalized coordinate derivative is constant. Examples are given to validate the effectiveness of the proposed continuous coordinated tracking algorithms.
\end{abstract}

\section{INTRODUCTION}

Coordination of multi-agent systems has been extensively studied for the past two decades. One fundamental problem is the coordinated tracking problem with a time-varying global objective [2], [3]. The key idea behind coordinated tracking problem is to control a group of followers to track a timevarying global objective by using only local information. The coordinated tracking problem was introduced and studied in [4] and [5], where the followers were modeled as single integrators. The tracking errors were shown to be bounded in [4] and the neighbors' control inputs were used in [5]. Recently, [6] proposed a coordinated tracking algorithm using a variable structure approach. Both the cases of multiple single integrators and multiple double integrators were considered and the tracking errors were shown to converge to zero using the proposed coordinated tracking algorithms.

In this paper, instead of modeling the follower dynamics as single integrators or double integrators, we study the coordinated tracking problem of multiple Lagrange systems. Here, a Lagrange system is used to represent a mechanical system, including spacecraft formations, vehicles, robotic manipulators, and mobile robots. Nonlinear contraction analysis was introduced in [7] to study the stability of coordinated tracking of multiple Lagrange systems under varieties of communication topologies. The author of [8] focused on the leaderless consensus of multiple Lagrange systems, where the generalized coordinate derivatives of the followers were driven to zero. Passivity-based control was used in [9], where time-varying delays, limited communication rates and non-vanishing bounded disturbances were considered. The

The authors are with ACCESS Linnaeus Centre, School of Electrical Engineering, Royal Institute of Technology, Stockholm 10044, Sweden. Email: $\{$ ziyangm, dimos, kallej\}@kth.se. Corresponding author: Z. Meng. Tel. +46-722-839377.

This work has been supported in part by the Knut and Alice Wallenbers Foundation and the Swedish Research Council, and in part by EU HYCON

2 NoE. An extended version of this work was recently submitted to IEEE Transactions on Robotics [1]. influence of communication delays was studied in [10] and adaptive controllers were used to guarantee both leaderless synchronization and leader-following coordinated tracking. Finite-time coordinated tracking algorithms were proposed in [11], [12], where the directed communication topology was emphasized in [11] and the Lagrange dynamics were used to represent the attitudes of rigid bodies in [12]. The authors of [13] introduced a variable structure approach by using both the one-hop and two-hop neighbors' information to achieve coordinated tracking. In addition, the containment control with group dispersion and group cohesion behaviors was reconstructed for multiple Lagrange systems in [14], where the proposed algorithm was discontinuous in order to dominate the external disturbances.

Compared with the existing literature, the contributions of the current paper are twofold. First, the proposed zero-error coordinated tracking algorithm is continuous, therefore free of chattering phenomena. This extends the existing results [11], [13], [14], where discontinuous control algorithms were proposed that may result in implementation issues. To the best of our knowledge, it is the first algorithm guaranteeing both zero-error tracking and chattering-free input in solving coordinated tracking problem of multiple Lagrange systems. Second, in contrast to [12], where the eigenvalues of the interaction Laplacian matrix and the upper bound of states of the bounded time-varying leader are assumed to be available to all the followers, the proposed algorithm in the current paper is purely distributed in the sense that both the control input and coupling gain depend only on local information.

The remainder of the paper is organized as follows. In Section II, we formulate the problem of coordinated tracking of multiple Lagrange systems and give some basic notations and definitions. The main results are presented in Sections III and III-A. Numerical studies are carried out in Section IV to validate the theoretical results and a brief concluding remark is given in Section V.

\section{Problem Statement and Preliminaries}

\section{A. Problem Statement}

Suppose that there are $n$ follower agents in the group, labeled as agents 1 to $n$. In addition to the $n$ followers, there also exists a leader agent in the group, labeled as agent 0 with the desired time-varying generalized coordinate $q_{0} \in \mathbb{R}^{p}$ and the desired time-varying generalized coordinate derivative $\dot{q}_{0} \in \mathbb{R}^{p}$. The objective of this paper is to design continuous coordinated tracking algorithms for follows such that the states of followers converge to those of the leader by using only local interactions, i.e., the leader states $q_{0}$ and $\dot{q}_{0}$ are 


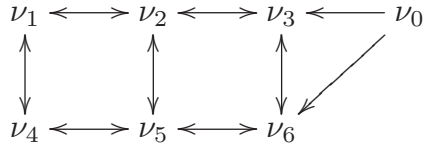

Fig. 1. Information flow associated with the leader and the six followers

only available to a subset of the followers and the followers only have access to their local neighbors' information.

In this paper, the system dynamics of the followers can be described by Lagrange equations

$$
M_{i}\left(q_{i}\right) \ddot{q}_{i}+C_{i}\left(q_{i}, \dot{q}_{i}\right) \dot{q}_{i}+g_{i}\left(q_{i}\right)=\tau_{i}, i=1,2, \cdots, n,
$$

where $q_{i} \in \mathbb{R}^{p}$ is the vector of generalized coordinates, $M_{i}\left(q_{i}\right) \in \mathbb{R}^{p \times p}$ is the $p \times p$ inertia (symmetric) matrix, $C_{i}\left(q_{i}, \dot{q}_{i}\right) \dot{q}_{i}$ is the Coriolis and centrifugal terms, $g_{i}\left(q_{i}\right)$ is the vector of gravitational forces, and $\tau_{i} \in \mathbb{R}^{p}$ is the control force. Note that (1) can be used to describe rigid bodies, robotic manipulators, and mobile robots. For example, we can transform attitude kinematics and dynamics of rigid bodies to their Lagrange expression using the relationship given in [15]. In general, the dynamics of a Lagrange system satisfies the following properties [16]:

1. $M_{i}\left(q_{i}\right)$ is positive definite.

2. $\dot{M}_{i}\left(q_{i}\right)-2 C_{i}\left(q_{i}, \dot{q}_{i}\right)$ is skew symmetric.

3. The left-hand side of the dynamics can be parameterized, i.e., $M_{i}\left(q_{i}\right) y+C_{i}\left(q_{i}, \dot{q}_{i}\right) x+g_{i}\left(q_{i}\right)=Y_{i}\left(q_{i}, \dot{q}_{i}, x, y\right) \theta_{i}$, $\forall x, y \in \mathbb{R}^{p}$, where $Y_{i} \in \mathbb{R}^{p \times p_{\theta}}$ is a regression matrix and $\theta_{i} \in \mathbb{R}^{p_{\theta}}$ is a constant vector identifying parameters of Lagrange dynamics.

In the real applications, the actual parameter $\theta_{i}$ may be not available. Instead, the nominal parameter $\widehat{\theta}_{i}$ is available. From Property 3, we know that the nominal dynamics satisfies

$$
\widehat{M}_{i}\left(q_{i}\right) \ddot{q}_{i}+\widehat{C}_{i}\left(q_{i}, \dot{q}_{i}\right) \dot{q}_{i}+\widehat{g}_{i}\left(q_{i}\right)=Y_{i}\left(q_{i}, \dot{q}_{i}, \dot{q}_{i}, \ddot{q}_{i}\right) \widehat{\theta}_{i},
$$

where $\widehat{M}_{i}\left(q_{i}\right), \widehat{C}_{i}\left(q_{i}, \dot{q}_{i}\right), \widehat{g}_{i}\left(q_{i}\right)$, and $\widehat{\theta}_{i}$ are nominal dynamics terms. We also know that the actual dynamics satisfies

$$
M_{i}\left(q_{i}\right) \ddot{q}_{i}+C_{i}\left(q_{i}, \dot{q}_{i}\right) \dot{q}_{i}+g_{i}\left(q_{i}\right)=Y_{i}\left(q_{i}, \dot{q}_{i}, \dot{q}_{i}, \ddot{q}_{i}\right) \theta_{i} .
$$

For later use, we define $\triangle \theta_{i}=\widehat{\theta}_{i}-\theta_{i}$. Considering that there are six followers $(n=6)$ in the group, Fig. 1 gives an example of information flow among the leader and six followers. Note that the leader's states are only available to followers $\nu_{3}$ and $\nu_{6}$ and the followers only have access to their neighbors' information.

\section{B. Graph Theory}

We use graphs to represent the communication topology among agents. A directed graph $\mathcal{G}_{n}$ consists of a pair $\left(\mathcal{V}_{n}, \mathcal{E}_{n}\right)$, where $\mathcal{V}_{n}=\{1,2, \ldots, n\}$ is a finite, nonempty set of nodes and $\mathcal{E}_{n} \subseteq \mathcal{V}_{n} \times \mathcal{V}_{n}$ is a set of ordered pairs of nodes. An edge $(i, j)$ denotes that node $j$ has access to the information from node $i$. An undirected graph is defined such that $(j, i) \in \mathcal{E}_{n}$ implies $(i, j) \in \mathcal{E}_{n}$. A directed path in a directed graph or an undirected path in an undirected graph is a sequence of edges of the form $(i, j),(j, k), \ldots$ The neighbors of node $i$ are defined as the set $N_{i}:=\{j \mid(j, i) \in$ $\left.\mathcal{E}_{n}\right\}$.

For a follower graph $\mathcal{G}_{n}$, its adjacency matrix $\mathcal{A}_{n}=$ $\left[a_{i j}\right] \in \mathbb{R}^{n \times n}$ is defined such that $a_{i j}$ is positive if $(j, i) \in \mathcal{E}_{n}$ and $a_{i j}=0$ otherwise. Here we assume that $a_{i i}=0$, $\forall i=1,2, \cdots, n$. The Laplacian matrix $\mathcal{L}_{n}=\left[l_{i j}\right] \in$ $\mathbb{R}^{n \times n}$ associated with $\mathcal{A}_{n}$ is defined as $l_{i i}=\sum_{j \neq i} a_{i j}$ and $l_{i j}=-a_{i j}$, where $i \neq j$. Similarly, we define the follower and leader graph $\mathcal{G}_{n+1}:=\left(\mathcal{V}_{n+1}, \mathcal{E}_{n+1}\right)$, where $\mathcal{V}_{n+1}=$ $\{0,1, \ldots, n\}, \mathcal{E}_{n+1} \subseteq \mathcal{V}_{n+1} \times \mathcal{V}_{n+1}$, and 0 denotes the leader and $1,2, \ldots, n$ denote the followers. The adjacency matrix $\mathcal{A}_{n+1}=\left[a_{i j}\right] \in \mathbb{R}^{(n+1) \times(n+1)}$ associated with $\mathcal{G}_{n+1}$ is defined such that $a_{i 0}$ is positive if $(0, i) \in \mathcal{E}_{n+1}$ and $a_{i 0}=0$ otherwise, $\forall i=1,2, \cdots, n$. Here we assume that $a_{i i}=0, \forall i$, and the leader has no parent, i.e., $, a_{0 j}=0, j=$ $0,1, \cdots, n$.

Assumption 1: The fixed undirected graph $\mathcal{G}_{n}$ is connected and $a_{i 0}>0$ for at least one $i, i=1,2, \cdots, n$.

Letting $\mathcal{M}=\mathcal{L}_{n}+\operatorname{diag}\left(a_{10}, a_{20}, \cdots, a_{n 0}\right)\left(\mathcal{L}_{n}\right.$ is the Laplacian matrix associated with $\mathcal{G}_{n}$ ), we recall the following result.

Lemma 1: [17] Under Assumption 1, $\mathcal{M}$ is symmetric and positive definite.

\section{Nonsmooth Analysis}

Consider the vector differential equation

$$
\dot{x}=f(x, t),
$$

where $f: \mathbb{R}^{p} \times \mathbb{R} \rightarrow \mathbb{R}^{p}$ is measurable and essentially locally bounded. A vector function $x(t)$ is called a solution of (2) on $\left[t_{0}, t_{1}\right]$ if $x(t)$ is absolutely continuous on $\left[t_{0}, t_{1}\right]$ and for almost all $t \in\left[t_{0}, t_{1}\right], \dot{x} \in \mathbb{K}[f](x, t)$ (see [19] for more details on the definition of $\mathbb{K}[f](x, t))$. Throughout this paper, the solutions to the closed-loop systems are understood in the Filippov sense.

For a locally Lipschitz function $V: \mathbb{R}^{p} \times \mathbb{R} \rightarrow \mathbb{R}$, the generalized gradient of $V$ at $(x, t)$ is defined by $\partial V(x, t)=$ $\overline{c o}\left\{\lim \nabla V(x, t) \mid\left(x_{i}, t_{i}\right) \rightarrow(x, t),\left(x_{i}, t_{i}\right) \notin \Omega_{V}\right\}$, where $\Omega_{V}$ is the set of measure zero where the gradient of $V$ is not defined. The generalized time derivative of $V$ with respect to (2) is defined as $\dot{\tilde{V}}:=\bigcap_{\zeta \in \partial V} \zeta^{\mathrm{T}}\left(\begin{array}{c}\mathbb{K}[f](x, t) \\ 1\end{array}\right)[18]$, [19], where $\zeta \in \partial V(x(t), t)$.

Lemma 2: [20] Let (2) be essentially locally bounded and $0 \in \mathbb{K}[f](x, t)$ in a region $\mathbb{R}^{p} \times[0, \infty)$. Furthermore, suppose that $f(0, t)$ is uniformly bounded for all $t \geq 0$. Let $V$ : $\mathbb{R}^{p} \times[0, \infty) \rightarrow \mathbb{R}$ be locally Lipschitz in $t$, and regular (see [19] for the definition of "regular") such that, $\forall t \geq 0$,

$$
\begin{gathered}
W_{1}(x) \leq V(x, t) \leq W_{2}(x), \\
\dot{\tilde{V}}(x, t) \leq-W(x),
\end{gathered}
$$

where $W_{1}(x)$ and $W_{2}(x)$ are continuous positive definite functions and $W(x)$ is a continuous positive semidefinite function. Then all the solutions of (2) are bounded and satisfy $W(x(t)) \rightarrow 0$, as $t \rightarrow \infty$. 


\section{Additional Notation}

Given a vector $x=\left[x_{1}, x_{1}, \cdots, x_{n}\right]^{\mathrm{T}}$, we define $\operatorname{sgn}(x)=\left[\operatorname{sgn}\left(x_{1}\right), \operatorname{sgn}\left(x_{2}\right), \cdots, \operatorname{sgn}\left(x_{n}\right)\right]^{\mathrm{T}}$, and $|x|=$ $\left[\left|x_{1}\right|,\left|x_{2}\right|, \cdots,\left|x_{n}\right|\right]^{\mathrm{T}}$. In addition, $\operatorname{diag}(x)$ denotes the diagonal matrix of a vector $x, \lambda_{\min }(P)$ and $\lambda_{\max }(P)$ denote respectively the minimum and maximum eigenvalues of the matrix $P$.

\section{ZERO-ERROR COORDINATED TRACKING USING CONTINUOUS CONTROL}

The objective here is to drive the states of the followers to converge to those of the leader. Note here that the leader's information is available to only a portion of the followers and we use nominal parameters of Lagrange dynamics. The control protocol is proposed for each follower,

$$
\tau_{i}=Y_{i}\left(q_{i}, \dot{q}_{i}, \dot{q}_{r i}, \ddot{q}_{r i}\right) \widehat{\theta}_{i}-\alpha_{i} s_{i}, \quad i=1,2, \cdots, n,
$$

where $Y_{i}$ is defined in Sections II-A and $\alpha_{i}>0$ is an arbitrary positive constant. In addition, the adaptive control term, the virtual reference trajectory, the leader's generalized coordinate derivative estimator, and the sliding surface are, respectively, given by

$$
\begin{gathered}
\dot{\hat{\theta}}_{i}=-\kappa Y_{i}^{\mathrm{T}}\left(q_{i}, \dot{q}_{i}, \dot{q}_{r i}, \ddot{q}_{r i}\right) s_{i} \\
\dot{q}_{r i}=\widehat{v}_{i}-b\left(\sum_{j=0}^{n} a_{i j}\left(q_{i}-q_{j}\right)\right) \\
\dot{\widehat{v}}_{i}(t)=-\left(k_{1}+1\right) \widehat{v}_{i}(t)-\int_{0}^{t}\left(k_{2 i}(\tau) \sum_{j=0}^{n} a_{i j}\left(\widehat{v}_{i}(\tau)-\widehat{v}_{j}(\tau)\right)\right. \\
+\beta_{i}(\tau) \operatorname{sgn}\left(\sum_{j=0}^{n} a_{i j}\left(\widehat{v}_{i}(\tau)-\widehat{v}_{j}(\tau)\right)\right) \mathrm{d} \tau
\end{gathered}
$$

and

$$
s_{i}=\dot{q}_{i}-\dot{q}_{r i},
$$

where $\widehat{v}_{0}=\dot{q}_{0}, a_{i j}$ is the $(i, j)$ th entry of $\mathcal{A}_{n+1}$ associated with $\mathcal{G}_{n+1}$ defined in Section II-B, $b>0, \kappa>0, k_{1}>0$ are arbitrary positive constants,

$$
\begin{aligned}
k_{2 i}(t)= & \frac{1}{2}\left(\sum_{j=0}^{n} a_{i j}\left(\widehat{v}_{i}(t)-\widehat{v}_{j}(t)\right)\right)^{\mathrm{T}}\left(\sum_{j=0}^{n} a_{i j}\left(\widehat{v}_{i}(t)-\widehat{v}_{j}(t)\right)\right) \\
& +\int_{0}^{t}\left(\sum_{j=0}^{n} a_{i j}\left(\widehat{v}_{i}(\tau)-\widehat{v}_{j}(\tau)\right)\right)^{\mathrm{T}} \\
& \times\left(\sum_{j=0}^{n} a_{i j}\left(\widehat{v}_{i}(\tau)-\widehat{v}_{j}(\tau)\right)\right) \mathrm{d} \tau
\end{aligned}
$$

and

$$
\begin{aligned}
\beta_{i}(t)= & \left\|\sum_{j=0}^{n} a_{i j}\left(\widehat{v}_{i}(t)-\widehat{v}_{j}(t)\right)\right\|_{1} \\
& +\int_{0}^{t}\left\|\sum_{j=0}^{n} a_{i j}\left(\widehat{v}_{i}(\tau)-\widehat{v}_{j}(\tau)\right)\right\|_{1} \mathrm{~d} \tau .
\end{aligned}
$$

Note that unlike the discontinuous algorithms given in [11], [13], [14], we introduce a continuous distributed estimator (6) to accurately obtain the leader's generalized coordinate derivative. The key idea here is to use a second-order sliding mode scheme instead of using a first-order sliding mode scheme. Before moving on, we need the following assumption and lemmas.

Assumption 2: $q_{0}$ is bounded up to its fourth derivative.

Lemma 3: [21] Let $\mathrm{S}$ be a symmetric matrix partitioned as $S=\left[\begin{array}{ll}S_{11} & S_{12} \\ S_{12}^{\mathrm{T}} & S_{22}\end{array}\right]$, where $S_{22}$ is square and nonsingular. Then $S>0$ if and only if $S_{22}>0$ and $S_{11}-S_{12} S_{22}^{-1} S_{12}^{\mathrm{T}}>0$.

Lemma 4: ([22], [23]) Define $\xi(t) \in \mathbb{R}^{p}$ as $\xi=(\mu+$ $\dot{\mu})^{\mathrm{T}}\left(-\bar{\beta} \operatorname{sgn}(\mu)+N_{d}\right)$, where $\mu(t) \in \mathbb{R}^{p}, \bar{\beta}$ is a positive constant, and $N_{d}(t) \in \mathbb{R}^{p}$ is the bounded disturbance. Then we have that $\int_{0}^{t} \xi(\tau) \mathrm{d} \tau \leq \mathcal{B}$, if $\bar{\beta}>\sup _{t}\left\|N_{d}(t)\right\|_{\infty}+$ $\sup _{t}\left\|\dot{N}_{d}(t)\right\|_{\infty}$, where $\mathcal{B}=\bar{\beta}\|\mu(0)\|_{1}-\mu^{\mathrm{T}}(0) N_{d}(0)>0$.

Theorem 1: Let Assumptions 1 and 2 hold. Under the local continuous coordinated tracking algorithm (3), the states of the followers governed by the Lagrange dynamics (1) globally asymptotically converge to those of the leader, i.e., $q_{i}(t) \rightarrow q_{0}(t)$ and $\dot{q}_{i}(t) \rightarrow \dot{q}_{0}(t), \forall q_{i}(0) \in \mathbb{R}^{p}$, $\forall i=1,2, \cdots, n$, as $t \rightarrow \infty$.

Proof:

It follows from Property 3 in Section II-A that

$M_{i}\left(q_{i}\right) \ddot{q}_{r i}+C_{i}\left(\dot{q}_{i}, q_{i}\right) \dot{q}_{r i}+g_{i}\left(q_{i}\right)=Y_{i}\left(q_{i}, \dot{q}_{i}, \dot{q}_{r i}, \ddot{q}_{r i}\right) \theta_{i}$.

We then further have that

$$
M_{i}\left(q_{i}\right) \dot{s}_{i}+C_{i}\left(\dot{q}_{i}, q_{i}\right) s_{i}=Y_{i}\left(q_{i}, \dot{q}_{i}, \dot{q}_{r i}, \ddot{q}_{r i}\right) \triangle \theta_{i}-\alpha_{i} s_{i},
$$

where $\triangle \theta_{i}$ is given in Section II-A. It also follows from (6) that

$$
\begin{aligned}
\ddot{\bar{v}}_{i}= & -\left(k_{1}+1\right) \dot{\bar{v}}_{i}-k_{2 i}\left(\sum_{j=1}^{n} a_{i j}\left(\bar{v}_{i}-\bar{v}_{j}\right)+a_{i 0} \bar{v}_{i}\right) \\
& -\beta_{i} \operatorname{sgn}\left(\sum_{j=1}^{n} a_{i j}\left(\bar{v}_{i}-\bar{v}_{j}\right)+a_{i 0} \bar{v}_{i}\right)+N_{d i},
\end{aligned}
$$

where $\bar{v}_{i}=\widehat{v}_{i}-\dot{q}_{0}, N_{d i}=-\left(k_{1}+1\right) \ddot{q}_{0}-\dddot{q}_{0}$. It follows that

$$
\begin{aligned}
\ddot{\bar{v}}_{i}= & -\left(k_{1}+1\right) \dot{\bar{v}}_{i}-k_{2 i}\left(\sum_{j=1}^{n} m_{i j} \bar{v}_{j}\right) \\
& -\beta_{i} \operatorname{sgn}\left(\sum_{j=1}^{n} m_{i j} \bar{v}_{j}\right)+N_{d i},
\end{aligned}
$$

where $m_{i j}$ denotes the $(i, j)$ th entry of $\mathcal{M}$ defined in Section II-B. Note that the right-hand side of (10) is discontinuous. 
Since the signum function is measurable and locally essentially bounded, we can rewrite (10) in terms of differential inclusions as

$$
\begin{gathered}
\ddot{\bar{v}}_{i} \in{ }^{a . e \cdot} \mathbb{K}\left[-\left(k_{1}+1\right) \dot{\bar{v}}_{i}-k_{2 i}\left(\sum_{j=1}^{n} m_{i j} \bar{v}_{j}\right)\right. \\
\left.-\beta_{i} \operatorname{sgn}\left(\sum_{j=1}^{n} m_{i j} \bar{v}_{j}\right)+N_{d i}\right],
\end{gathered}
$$

where a.e. stands for "almost everywhere".

Define $\eta_{i}=\bar{v}_{i}+\dot{\bar{v}}_{i}, \bar{v}=\left[\bar{v}_{1}^{\mathrm{T}}, \bar{v}_{2}^{\mathrm{T}}, \cdots, \bar{v}_{n}^{\mathrm{T}}\right]$, and $\eta=$ $\left[\eta_{1}^{\mathrm{T}}, \eta_{2}^{\mathrm{T}}, \cdots, \eta_{n}^{\mathrm{T}}\right]$. We construct a Lyapunov function candidate as:

$$
\begin{aligned}
V= & \frac{1}{2} \sum_{i=1}^{n} s_{i}^{T} M_{i}\left(q_{i}\right) s_{i}+\frac{1}{2 \kappa} \sum_{i=1}^{n}\left(\triangle \theta_{i}\right)^{\mathrm{T}} \triangle \theta_{i} \\
& +\frac{1}{2} \eta^{\mathrm{T}}\left(\mathcal{M} \otimes I_{p}\right) \eta+\frac{1}{2} \bar{k} \bar{v}^{\mathrm{T}}\left(\mathcal{M}^{2} \otimes I_{p}\right) \bar{v} \\
& +\frac{1}{2} \sum_{i=1}^{n}\left(k_{2 i}-\bar{k}\right)^{2}+\frac{1}{2} \sum_{i=1}^{n}\left(\beta_{i}-\bar{\beta}\right)^{2}+V_{0}
\end{aligned}
$$

where $V_{0}=\sum_{i=1}^{n} \mathcal{B}_{i}-\sum_{i=1}^{n} \int_{0}^{t}\left(\sum_{j=1}^{n} m_{i j} \eta_{j}(\tau)\right)^{\mathrm{T}} \times$ $\left(-\bar{\beta}_{\operatorname{sgn}}\left(\sum_{j=0}^{n} a_{i j}\left(\widehat{v}_{i}(\tau)-\widehat{v}_{j}(\tau)\right)\right)+N_{d i}(\tau)\right) \mathrm{d} \tau, \mathcal{B}_{i}=$ $\bar{\beta}\left\|\sum_{j=1}^{n} m_{i j} \eta_{j}(0)\right\|_{1}-\left(\sum_{j=1}^{n} m_{i j} \eta_{j}(0)\right)^{\mathrm{T}} N_{d i}(0)$. In addition, we select $\bar{\beta}$ and $\bar{k}$ as two positive constants satisfying that $\bar{\beta}>\sup _{t}\left\{\left(k_{1}+1\right)\left\|\ddot{q}_{0}(t)\right\|_{\infty}+\left(k_{1}+2\right)\left\|\dddot{q}_{0}(t)\right\|_{\infty}+\right.$ $\left.\left\|\dddot{q}_{0}(t)\right\|_{\infty}\right\}$ and $\bar{k}>\frac{k_{1}}{4 \lambda_{\min }(\mathcal{M})}$. It follows from Lemma 4 that $V_{0}>0$ when $\bar{\beta}>\sup _{t}\left\{\left(k_{1}+1\right)\left\|\ddot{q}_{0}(t)\right\|_{\infty}+\left(k_{1}+\right.\right.$ 2) $\left.\left\|\dddot{q}_{0}(t)\right\|_{\infty}+\left\|\dddot{q}_{0}(t)\right\|_{\infty}\right\}$. It follows that the generalized time derivative of $V$ can be evaluated as

$$
\begin{aligned}
\dot{\tilde{V}}= & \bigcap_{\xi \in \partial\|\mu\|_{1}}-\left(\left(\mathcal{M} \otimes I_{p}\right) \eta\right)^{\mathrm{T}}\left(-\bar{\beta} \xi+N_{d}\right) \\
& +\mathbb{K}\left[\sum_{i=1}^{n} s_{i}^{\mathrm{T}}\left(Y_{i}\left(q_{i}, \dot{q}_{i}, \dot{q}_{r i}, \ddot{q}_{r i}\right) \triangle \theta_{i}-\alpha_{i} s_{i}\right)\right. \\
& -\sum_{i=1}^{n}\left(\triangle \theta_{i}\right)^{\mathrm{T}} Y_{i}^{\mathrm{T}}\left(q_{i}, \dot{q}_{i}, \dot{q}_{r i}, \ddot{q}_{r i}\right) s_{i} \\
& +\sum_{i=1}^{n}\left(\sum_{j=1}^{n} m_{i j} \eta_{j}\right)^{\mathrm{T}}\left(-k_{2 i} \sum_{j=1}^{n} m_{i j} \bar{v}_{j}\right. \\
& \left.-k_{1} \dot{\bar{v}}_{i}-k_{1} \bar{v}_{i}+k_{1} \bar{v}_{i}-\beta_{i} \mathrm{sgn}\left(\sum_{j=1}^{n} m_{i j} \bar{v}_{j}\right)+N_{d i}\right) \\
& +\bar{k}_{\bar{v}}^{\mathrm{T}}\left(\mathcal{M}^{2} \otimes I_{p}\right)(\eta-\bar{v})+\sum_{i=1}^{n}\left(k_{2 i}-\bar{k}\right) \\
& \times\left(\sum_{j=1}^{n} m_{i j} \bar{v}_{j}\right)^{\mathrm{T}}\left(\sum_{j=1}^{n} m_{i j} \eta_{j}\right)+\sum_{i=1}^{n}\left(\beta_{i}-\bar{\beta}\right) \\
& \left.\times\left(\sum_{j=1}^{n} m_{i j} \eta_{j}\right)^{\mathrm{T}} \operatorname{sgn}\left(\sum_{j=1}^{n} m_{i j} \bar{v}_{j}\right)\right]
\end{aligned}
$$

where $N_{d}=\left[N_{d 1}^{\mathrm{T}}, N_{d 2}^{\mathrm{T}}, \ldots, N_{d n}^{\mathrm{T}}\right]^{\mathrm{T}}, \mu=\left(\mathcal{M} \otimes I_{p}\right) \bar{v}$, $\partial\left|\mu_{k}\right|=\left\{\begin{array}{l}\{-1\}, \quad \mu_{k} \in \mathbb{R}^{-} \\ \{1\}, \quad \mu_{k} \in \mathbb{R}^{+} \\ {[-1,1], \quad \mu_{k}=0,}\end{array} \quad\right.$ and $\mu_{k}$ is $k$ th entry of $\mu$.

We then have that

$$
\begin{aligned}
\dot{\tilde{V}}= & -k_{1} \eta^{\mathrm{T}}\left(\mathcal{M} \otimes I_{p}\right) \eta+k_{1} \eta^{\mathrm{T}}\left(\mathcal{M} \otimes I_{p}\right) \bar{v} \\
& -\bar{k} \bar{v}^{\mathrm{T}}\left(\mathcal{M}^{2} \otimes I_{p}\right) \bar{v}-\sum_{i=1}^{n} \alpha_{i} s_{i}^{\mathrm{T}} s_{i} \\
= & -\left[\begin{array}{ll}
\eta & \bar{v}
\end{array}\right]\left[\begin{array}{cc}
k_{1} \mathcal{M} \otimes I_{p} & -\frac{k_{1} \mathcal{M} \otimes I_{p}}{2} \\
-\frac{k_{1} \mathcal{M} \otimes I_{p}}{2} & \bar{k} \mathcal{M}^{2} \otimes I_{p}
\end{array}\right]\left[\begin{array}{c}
\eta \\
\bar{v}
\end{array}\right] \\
& -\sum_{i=1}^{n} \alpha_{i} s_{i}^{\mathrm{T}} s_{i} \\
= & -W(\eta, \bar{v}, s),
\end{aligned}
$$

where $s=\left[s_{1}, s_{2}, \ldots, s_{n}\right]^{\mathrm{T}}$. It follows from Lemma 3 that $\left[\begin{array}{cc}k_{1} \mathcal{M} \otimes I_{p} & -\frac{k_{1} \mathcal{M} \otimes I_{p}}{2} \\ -\frac{k_{1} \mathcal{M} \otimes I_{p}}{2} & \bar{k} \mathcal{M}^{2} \otimes I_{p}\end{array}\right]>0$ when $\bar{k}>\frac{k_{1}}{4 \lambda_{\min }(\mathcal{M})}$. This implies that $W(\eta, \bar{v}, s) \geq 0$ and therefore $V$ is bounded. Thus, $s_{i}, \triangle \theta_{i}, \bar{v}$, and $\eta$ are bounded. Note that the sliding surface $s_{i}$ can be rewritten as $\dot{\bar{q}}_{i}=-b \sum_{j=1}^{n} m_{i j} \bar{q}_{j}+s_{i}+\bar{v}_{i}$. This can be further written in the matrix form

$$
\dot{\bar{q}}=-\left(b \mathcal{M} \otimes I_{p}\right) \bar{q}+s+\bar{v} .
$$

Since $\mathcal{M}$ is positive definite, we know that (11) is input-tostate stable by considering $s+\bar{v}$ as the input. Therefore, it follows that $\bar{q}_{i}$ and $\dot{\bar{q}}_{i}, \forall i=1,2, \ldots, n$, are bounded based on the facts that $s$ and $\bar{v}$ are bounded. Then, we know that $q_{i}$ and $\dot{q}_{i}, \dot{q}_{r i}$ and $\ddot{q}_{r i}$ are bounded. This shows that $\dot{s}_{i}$ and $\dot{\eta}_{i}$ are bounded. It follows that $s_{i}(t), \eta_{i}(t)$, and $\bar{v}_{i}(t), \forall i=$ $1,2, \ldots, n$ are uniformly continuous in $t$. This shows that $W(\eta(t), \bar{v}(t), s(t))$ is uniformly continuous in $t$. Therefore, we know from Lemma 2 that $W(\eta(t), \bar{v}(t), s(t)) \rightarrow 0$, as $t \rightarrow \infty$. This shows that $\eta(t) \rightarrow 0, \bar{v}(t) \rightarrow 0$, and $s_{i}(t) \rightarrow 0$, as $t \rightarrow \infty$. Then, on the sliding surface $s_{i}=0$, we have that $\dot{q}_{i}-\dot{q}_{0}=-b \sum_{j=0}^{n} a_{i j}\left(q_{i}-q_{j}\right)$. Therefore we can easily show that $\dot{q}_{i}(t) \rightarrow \dot{q}_{0}(t)$ and $q_{i}(t) \rightarrow q_{0}(t), \forall i=1,2, \ldots, n$ as $t \rightarrow \infty$.

\section{A. Special Case: Coordinated Tracking When the Leader's Generalized Coordinate Derivative is Constant}

In this section, we consider a special case when $\dot{q}_{0}$ is constant. Therefore, $\ddot{q}_{0}=0$. The continuous control protocol proposed in algorithm (3) is considered again. The adaptive control term, the virtual reference trajectory, the sliding surface are given in (4), (5), and (7). In addition, the leader's generalized coordinate derivative estimator is proposed as

$$
\dot{\widehat{v}}_{i}=-\left(\sum_{j=1}^{n} a_{i j}\left(q_{i}-q_{j}\right)+a_{i 0}\left(q_{i}-q_{0}\right)\right),
$$

where $a_{i j}$ is the $(i, j)$ th entry of $\mathcal{A}_{n+1}$ associated with $\mathcal{G}_{n+1}$ defined in Section II-B.

Before moving on, we need the following assumption to proceed. 
Assumption 3: $M_{i}\left(q_{i}\right), C_{i}\left(q_{i}, \dot{q}_{i}\right)$, and $g_{i}\left(q_{i}\right)$ are continuously differentiable.

Theorem 2: Let Assumptions 1 and 3 hold. Under the local continuous coordinated tracking algorithm (3)-(5), (7) and (12), the states of the followers governed by the Lagrange dynamics (1) globally asymptotically converge to those of the leader, i.e., $q_{i}(t) \rightarrow q_{0}(t)$ and $\dot{q}_{i}(t) \rightarrow \dot{q}_{0}(t)$, $\forall q_{i}(0) \in \mathbb{R}^{p}, \forall i=1,2, \ldots, n$, as $t \rightarrow \infty$.

Proof:

It follows from Property 3 of Lagrange dynamics in Section II-A that $M_{i}\left(q_{i}\right) \ddot{q}_{r i}+C_{i}\left(\dot{q}_{i}, q_{i}\right) \dot{q}_{r i}+g_{i}\left(q_{i}\right)=$ $Y_{i}\left(q_{i}, \dot{q}_{i}, \dot{q}_{r i}, \ddot{q}_{r i}\right) \theta_{i}, \quad i=1,2, \ldots, n$. We then further have that

$$
\begin{array}{r}
M_{i}\left(q_{i}\right) \dot{s}_{i}+C_{i}\left(\dot{q}_{i}, q_{i}\right) s_{i}=Y_{i}\left(q_{i}, \dot{q}_{i}, \dot{q}_{r i}, \ddot{q}_{r i}\right) \triangle \theta_{i}-\alpha_{i} s_{i} \\
i=1,2, \ldots, n .
\end{array}
$$

We then construct a Lyapunov function candidate as $V=$ $\frac{1}{2} \sum_{i=1}^{n} s_{i}^{T} M_{i}\left(q_{i}\right) s_{i}+\sum_{i=1}^{n} \frac{1}{2 \kappa_{i}}\left(\triangle \theta_{i}\right)^{\mathrm{T}} \triangle \theta_{i}$.

Taking the derivative of $V$ along (13), we have that $\dot{V}=\sum_{i=1}^{n} s_{i}^{\mathrm{T}}\left(Y_{i}\left(q_{i}, \dot{q}_{i}, \dot{q}_{r i}, \ddot{q}_{r i}\right) \triangle \theta_{i}-\alpha_{i} s_{i}\right)-$ $\sum_{i=1}^{n}\left(\triangle \theta_{i}\right)^{\mathrm{T}} Y_{i}^{\mathrm{T}}\left(q_{i}, \dot{q}_{i}, \dot{q}_{r i}, \ddot{q}_{r i}\right) s_{i}=-\sum_{i=1}^{n} \alpha_{i} s_{i}^{\mathrm{T}} s_{i} \leq 0$, where we have used Property 2 of Lagrange dynamics in Section II-A. It follows that $V$ is bounded. We then know that $s_{i}$ and $\triangle \theta_{i}, \forall i=1,2, \ldots, n$, are bounded from Property 1 of Lagrange dynamics in Section II-A. Therefore, it follows that $q_{i}, \dot{q}_{i}, \dot{q}_{r i}$, and $\ddot{q}_{r i}, \forall i=1,2, \ldots, n$, are bounded. This shows that $\dot{s}_{i}, \forall i=1,2, \ldots, n$, is bounded and thus $\ddot{V}$ is bounded. It then follows from Barbalat's lemma that $\dot{V} \rightarrow 0$, as $t \rightarrow \infty$. Since $\dot{s}_{i}$ is bounded, we also know that $\ddot{q}_{i}$ and $\dddot{q}_{r i}$ are bounded. It then follows from (1) with (3)-(5), (7) and (12) and Assumption 3 that $M_{i}\left(q_{i}\right) \dddot{q}_{i}+\dot{M}_{i}\left(q_{i}\right) \ddot{q}_{i}+$ $C_{i}\left(\dot{q}_{i}, q_{i}\right) \ddot{q}_{i}+\dot{C}_{i}\left(\dot{q}_{i}, q_{i}\right) \dot{q}_{i}+\dot{g}_{i}\left(q_{i}\right)=\widehat{M}_{i}\left(q_{i}\right) \dddot{q}_{i r}+\dot{\widehat{M}}_{i}\left(q_{i}\right) \ddot{q}_{i r}+$ $\widehat{C}_{i}\left(\dot{q}_{i}, q_{i}\right) \ddot{q}_{i r}+\dot{\widehat{C}}_{i}\left(\dot{q}_{i}, q_{i}\right) \dot{q}_{i r}+\dot{\widehat{g}}_{i}\left(q_{i}\right)-\alpha_{i} \dot{s}_{i}$.

Therefore, we know that $\dddot{q}_{i}$ is bounded and thus $\ddot{s}_{i}$ is bounded. It then follows from Barbalat's lemma that $\dot{s}_{i}(t) \rightarrow$ 0 , as $t \rightarrow \infty$. Also note that $\ddot{q}_{i}=-b\left(\sum_{j=0}^{n} a_{i j}\left(\dot{q}_{i}-\dot{q}_{j}\right)\right)-$ $\left(\sum_{j=0}^{n} a_{i j}\left(q_{i}-q_{j}\right)\right)+\dot{s}_{i}$. This can be further written as

$$
\ddot{\bar{q}}_{i}=-b \sum_{j=1}^{n} m_{i j} \dot{\bar{q}}_{j}-\sum_{j=1}^{n} m_{i j} \bar{q}_{j}+\dot{s}_{i},
$$

where $\bar{q}_{i}=q_{i}-q_{0}, \forall i=1,2, \ldots, n$, and $m_{i j}$ denotes the $(i, j)$ th entry of $\mathcal{M}$ defined in Section II-B. Considering the closed-loop system $\ddot{\bar{q}}_{i}=-\sum_{j=1}^{n} m_{i j} \bar{q}_{j}-b \sum_{j=1}^{n} m_{i j} \dot{\bar{q}}_{j}$, we construct the following Lyapunov function candidate as,

$$
V_{2}=\frac{1}{2} \sum_{i=1}^{n} \dot{\bar{q}}_{i}^{\mathrm{T}} \dot{\bar{q}}_{i}+\frac{1}{2} q^{\mathrm{T}}\left(\mathcal{M} \otimes I_{p}\right) q,
$$

where $q=\left[q_{1}^{\mathrm{T}}, q_{2}^{\mathrm{T}}, \ldots, q_{n}^{\mathrm{T}}\right]^{\mathrm{T}}$. Note that $\mathcal{M}$ is positive definite from Lemma 1 if Assumption 1 is satisfied. It is then trivial to show that $\bar{q}_{i}=0$ and $\dot{\bar{q}}_{i}=0, \forall i=$ $1,2, \ldots, n$, are globally asymptotically equilibrium points for $\ddot{\bar{q}}_{i}=-\sum_{j=1}^{n} m_{i j} \bar{q}_{j}-b \sum_{j=1}^{n} m_{i j} \dot{\bar{q}}_{j}, i=1,2, \ldots, n$. Combing the fact that $\dot{s}_{i}(t) \rightarrow 0$, as $t \rightarrow \infty$, we can show that $\bar{q}_{i}(t) \rightarrow 0$ and $\dot{\bar{q}}_{i}(t) \rightarrow 0, \forall i=1,2, \ldots, n$, as $t \rightarrow \infty$, that is, $q_{i}(t) \rightarrow q_{0}(t)$ and $\dot{q}_{i}(t) \rightarrow \dot{q}_{0}(t), \forall q_{i}(0) \in \mathbb{R}^{p}$, $\forall i=1,2, \ldots, n$, as $t \rightarrow \infty$.

\section{Simulation Results}

In this section, numerical simulation results are given to validate the effectiveness of the theoretical results obtained in this paper. We assume that there exist $n=6$ followers. The system dynamics of the followers are given by the Lagrange dynamics of the two-link manipulators [13], [16], [24],

$$
\begin{aligned}
{\left[\begin{array}{cc}
M_{11, i} & M_{12, i} \\
M_{21, i} & M_{22, i}
\end{array}\right] } & {\left[\begin{array}{c}
\ddot{q}_{i x} \\
\ddot{q}_{i y}
\end{array}\right]+\left[\begin{array}{ll}
C_{11, i} & C_{12, i} \\
C_{21, i} & C_{22, i}
\end{array}\right]\left[\begin{array}{c}
\dot{q}_{i x} \\
\dot{q}_{i y}
\end{array}\right] } \\
& +\left[\begin{array}{c}
g_{1, i} \\
g_{2, i}
\end{array}\right]=\left[\begin{array}{c}
\tau_{i x} \\
\tau_{i y}
\end{array}\right], i=1,2, \ldots, 6,
\end{aligned}
$$

where $M_{11, i}=\theta_{1 i}+2 \theta_{2 i} \cos q_{i y}, M_{12, i}=M_{21, i}=\theta_{3 i}+$ $\theta_{2 i} \cos q_{i y}, M_{22, i}=\theta_{3 i}, C_{11, i}=-\theta_{2 i} \sin q_{i y} \dot{q}_{i y}, C_{12, i}=$ $-\theta_{2 i} \sin q_{i y}\left(\dot{q}_{i x}+\dot{q}_{i y}\right), C_{21, i}=\theta_{2 i} \sin q_{i y} \dot{q}_{i x}, C_{22, i}=0$, $g_{1, i}=\theta_{4 i} g \cos q_{i x}+\theta_{5 i} g \cos \left(q_{i x}+q_{i y}\right), g_{2, i}=\theta_{5 i} g \cos \left(q_{i x}+\right.$ $\left.q_{i y}\right)$ and $g=9$.8. Also, $\theta_{1 i}=m_{1 i} l_{c 1, i}^{2}+m_{2 i}\left(l_{1 i}^{2}+l_{c 2, i}^{2}\right)+$ $J_{1 i}+J_{2 i}, \theta_{2 i}=m_{2 i} l_{1 i} l_{c 2, i}, \theta_{3 i}=m_{2 i} l_{c 2, i}^{2}+J_{2 i}, \theta_{4 i}=$ $m_{1 i} l_{c 1, i}+m_{2 i} l_{1 i}, \theta_{5 i}=m_{2 i} l_{2 i}$. We choose $m_{1 i}=1+0.3 i$, $m_{2 i}=1.5+0.3 i, l_{l i}=0.2+0.06 i, l_{2 i}=0.3+0.06 i, l_{c 1, i}=$ $0.1+0.03 i, l_{c 2, i}=0.15+0.03 i, J_{1 i}=\frac{m_{1 i} l_{l i}^{2}}{12}, J_{2 i}=\frac{m_{2 i} l_{2 i}^{2}}{12}$, $i=1,2, \ldots, 6$.

According to property 3 of Lagrange dynamics given in Section II-A, the dynamics of the followers can be parameterized as $Y_{i}\left(q_{i}, \dot{q}_{i}, \dot{q}_{r i}, \ddot{q}_{r i}\right)=\left[y_{p q}\right]_{i} \in \mathbb{R}^{2 \times 5}$, where $\theta_{i}=\left[\theta_{1 i}, \theta_{2 i}, \theta_{i 3}, \theta_{i 4}, \theta_{i 5}\right]^{\mathrm{T}}, y_{11}=\ddot{q}_{r i, x}, y_{12}=\left(2 \ddot{q}_{r i, x}+\right.$ $\left.\ddot{q}_{r i, y}\right) \cos q_{i y}-\left(\dot{q}_{i y} \dot{q}_{r i, x}+\dot{q}_{i x} \dot{q}_{r i, y}+\dot{q}_{i y} \dot{q}_{r i, y}\right) \sin q_{i y}, y_{13}=$ $\ddot{q}_{r i, y}, y_{14}=g \cos q_{i x}, y_{15}=g \cos \left(q_{i x}+q_{i y}\right), y_{21}=0$, $y_{22}=\ddot{q}_{r i, x} \cos q_{i y}+\dot{q}_{i x} \dot{q}_{r i, x} \sin q_{i y}, y_{23}=\ddot{q}_{r i, x}+\ddot{q}_{r i, y}$, $y_{24}=0, y_{25}=g \cos \left(q_{i x}+q_{i y}\right)$.

The initial states of the followers are given by $q_{i x}(0)=$ $0.6 i, q_{i y}(0)=0.4 i-1, \dot{q}_{i x}(0)=0.05 i-0.2, \dot{q}_{i y}(0)=$ $-0.05 i+0.2, i=1,2, \ldots, 6$. The leader-follower communication topology is given in Fig. 1. The adjacency matrix $\mathcal{A}_{n}$ of the generalized coordinate derivatives associated with $\mathcal{G}_{n}$ is chosen to be $\mathcal{A}_{n}=\left[\begin{array}{llllll}0 & 1 & 0 & 1 & 0 & 0 \\ 1 & 0 & 1 & 0 & 1 & 0 \\ 0 & 1 & 0 & 0 & 0 & 1 \\ 1 & 0 & 0 & 0 & 1 & 0 \\ 0 & 1 & 0 & 1 & 0 & 1 \\ 0 & 0 & 1 & 0 & 1 & 0\end{array}\right]$, and $a_{10}=0, a_{20}=0, a_{30}=1, a_{40}=0, a_{50}=0, a_{60}=1$. The initial estimations for $\theta_{1 i}, \theta_{2 i}, \theta_{3 i}, \theta_{4 i}$, and $\theta_{5 i}$ for each follower $i=1,2, \ldots, 6$, are given by $\hat{\theta}_{1 i}(0)=0, \hat{\theta}_{2 i}(0)=0$, $\widehat{\theta}_{3 i}(0)=0, \widehat{\theta}_{4 i}(0)=0$, and $\widehat{\theta}_{5 i}(0)=0$.

For the case of coordinated tracking when the leader's generalized coordinate derivative is time-varying (algorithm (3)), the trajectories of the leader are given by $q_{0 x}(t)=\cos \left(\frac{\pi}{15} t\right)$ and $q_{0 y}(t)=\sin \left(\frac{\pi}{15} t\right)$. The constant control parameters are chosen by $b=1, \kappa=2, k_{1}=0.5, \alpha_{i}=1, \forall i=$ $1,2, \ldots, 6$. The initial states of $k_{2 i}$ and $\beta_{i}$ for each follower $i=1,2, \ldots, 6$ are given by $k_{2 i}(0)=0$ and $\beta_{i}(0)=0$. The initial states of $\widehat{v}_{i}$ for each follower $i=1,2, \ldots, 6$ are given by $\widehat{v}_{i}(0)=\dot{\widehat{v}}_{i}(0)=[0,0]^{\mathrm{T}}$. Under the feedback algorithm (3), the generalized coordinates, the generalized coordinate 



(a) The trajectories of the states and the control torques of the followers and the leader
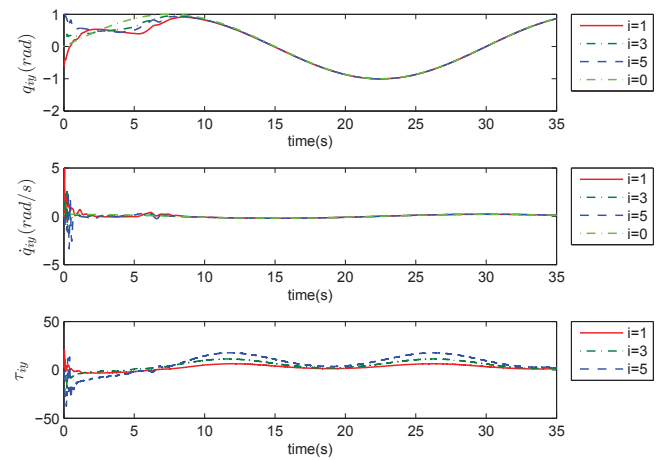

(b) The trajectories of the states and the control torques of the followers and the leader

Fig. 2. The states and the control torques of system (1) under algorithm (3)

derivatives, and the control torques of the followers and the leader are shown in Figs. 2(a) and 2(b). We see that the coordinated tracking is achieved for a group of Lagrange systems when the leader's generalized coordinate derivative is time-varying.

\section{CONCluding REMARKS}

In this paper, a continuous coordinated tracking algorithm was proposed for a group of Lagrange systems. We showed that the states of the followers were driven to converge to those of the leader and the tracking errors between the followers and the leader converge to zero. Then, we extended the continuous coordinated tracking algorithm to the case when the leader's generalized coordinate derivative is constant. Simulations were given to validate the effectiveness of the proposed algorithms. Further direction includes the study of switching communication topology for the coordinated tracking problem of multiple Lagrange systems.

\section{REFERENCES}

[1] Z. Meng, D. V. Dimarogonas, and K. H. Johansson, "Leader-follower coordinated tracking of multiple heterogeneous Lagrange systems using continuous control algorithms," IEEE Transations on Robotics, submitted.
[2] W. Ren, R. W. Beard, and E. M. Atkins, "Information consensus in multivehicle cooperative control: Collective group behavior through local interaction," IEEE Control Systems Magazine, vol. 27, no. 2, pp. 71-82, 2007.

[3] R. Olfati-Saber, J. A. Fax, and R. M. Murray, "Consensus and cooperation in networked multi-agent systems," Proceedings of the IEEE, vol. 95, no. 1, pp. 215-233, 2007.

[4] Y. Hong, J. Hu and L. Gao, "Tracking control for multi-agent consensus with an active leader and variable topology," Automatica, vol. 42, no. 7, pp. 1177-1182, 2007.

[5] W. Ren, "Multi-vehicle consensus with a time-varying reference state," Systems \& Control Letters, vol. 56, no. 7-8, pp. 474-483, 2007.

[6] Y. Cao and W. Ren, "Distributed coordinated tracking with reduced interaction via a variable structure approach," IEEE Transactions on Automatic Control, vol. 57, no. 1, pp. 33-48, 2012.

[7] S. J. Chung and J. J. E. Slotine, "Cooperative robot control and concurrent synchronization of Lagrangian systems," IEEE Transations on Robotics, vol. 25, no. 3, pp. 686-700, 2009.

[8] W. Ren, "Distributed leaderless consensus algorithms for networked Euler-Lagrange systems," International Journal of Control, vol. 82 no. 11, pp. 2137-2149, 2009.

[9] P. F. Hokayem, D. M. Stipanovic and M. W. Spong, "Semiautonomous control of multiple networked Lagrangian systems," International Journal of Robust and Nonlinear Control, vol. 19, no. 18, pp. 20402055, 2008

[10] E. Nuno, R. Ortega, L. Basanez and D. Hill, "Synchronization of networks of nonidentical Euler-Lagrange systems with uncertain parameters and communication delays," IEEE Transcations on Automatic Control, vol. 56, no. 4, pp. 935-941, 2011.

[11] S. Khoo, L. Xie, and Z. Man, "Robust finite-time consensus tracking algorithm for multirobot systems," IEEE/ASME Transcations on Mechatronics, vol. 14, no. 2, pp. 219-228, 2009.

[12] Z. Meng, W. Ren, and Z. You, "Distributed finite-time attitude containment control for multiple rigid bodies," Automatica, vol. 46 , no. 12, pp. 2092-2099, 2010.

[13] J. Mei and W. Ren, "Distributed coordinated tracking with a dynamic leader for multiple Euler-Lagrange systems," IEEE Transcations on Automatic Control, vol. 56, no. 6, pp. 1415-1421, 2011.

[14] Z. Meng, Z. Lin and W. Ren, "Leader-follower swarm tracking for networked Lagrange systems," Systems \& Control Letters, vol. 61, no. 1 , pp. 117-126, 2012

[15] J.-J. E. Slotine, and M. D. D. Benedetto, "Hamiltonian adaptive control of spacecraft", IEEE Transcations on Automatic Control, vol. 35, no. 7, pp. 848-852, 1990.

[16] M. W. Spong, S. Hutchinson, and M. Vidyasagar, Robot Modeling and Control, John Wiley \& Sons, Inc., 2006.

[17] J. Hu and Y. Hong, "Leader-following coordination of multi-agent systems with coupling time delays," Physica A: Statistical Mechanics and its Applications, vol. 374, no. 2, pp. 853-863, 2007.

[18] B. E. Paden and S. S. Sastry, "A calculus for computing Filippov's differential inclusion with application to the variable structure control of robot manipulators," IEEE Transactions on Circuits and Systems, vol. 34, no. 1, pp. 73-82, 1987.

[19] D. Shevitz and B. Paden, "Lyapunov stability theory of nonsmooth systems," IEEE Transcations on Automatic Control, vol. 39, no. 9, pp. 1910-1914, 1994.

[20] N. Fischer, R. Kamalapurkar and W. E. Dixon, "Lasalle-Yoshizawa corollaries for nonsmooth systems," IEEE Transcations on Automatic Control, vol. 58, no. 9, pp. 2333-2338, 2013.

[21] S. P. Boyd, L. E. Ghaoui, E. Feron, V. Balakrishnan, Linear Matrix Inequalities in System and Control Theory, SIAM, Philadelphia, 1994.

[22] B. Xian, D. M. Dawson, M. S. de Queiroz and J. Chen, "A continuous asymptotic tracking control strategy for uncertain nonlinear systems," IEEE Transactions on Automatic Control, vol. 49, no. 7, pp. 12061211,2004

[23] B. Xian, M. S. de Queiroz, D. M. Dawson, and M. L. McIntyre, "A discontinuous output feedback controller and velocity observer for nonlinear mechanical systems," Automatica, vol. 40, no. 4, pp. 695 700, 2004.

[24] H. Min, F. Sun, S. Wang, and H. Li, "Distributed adaptive consensus algorithm for networked Euler-Lagrange systems," IET Control Theory and Applications, vol. 5, no. 1, pp. 145-154, 2011. 